\title{
ON THE SEMI-CANONICAL PROPERTY IN THE PRODUCT SPACE $X \times I$
}

\author{
A. OKUYAMA AND Y. YASUI
}

\begin{abstract}
As one of the several properties in generalized metric spaces, the semi-canonical property has been discussed from the viewpoint of the extension of mappings. In this paper, that property will be discussed in product space $X \times I$ and reduced to a property of $X$.
\end{abstract}

1. Introduction. By a pair $(X, A)$ we mean a topological space $X$ with a closed subset $A$ of $X$. Let $(X, A)$ be a pair. As in [6], a collection $\mathcal{V}=\left\{V_{\lambda}\right.$ : $\lambda \in \Lambda$ \} of open subsets of $X$ is called a semi-canonical cover for $(X, A)$ if

(1) $\cup_{\lambda \in \Lambda} V_{\lambda}=X-A$, and

(2) for each $x \in A$ and each neighborhood $U$ of $x$ in $X$ there exists a neighborhood $W$ of $x$ in $X$ such that $\operatorname{St}(W, \mathfrak{V}) \subset U$, where

$$
\operatorname{St}(W, \mathscr{V})=\cup\{V \in \mathscr{V}: V \cap W \neq \varnothing\}
$$

denotes the star of $W$ with respect to $\mathcal{T}$.

If a semi-canonical cover exists for $(X, A),(X, A)$ is called a semicanonical pair.

It was proved by D. Hyman ([6], [7]) that $(X, A)$ is a semi-canonical pair if $X$ is the image of a metric space by a closed continuous map. It is also mentioned by M. Cauty [3] that, if $X$ is a stratifiable space (cf. [2]), then any pair $(X, A)$ is semi-canonical. However, quite recently S. San-ou [11] pointed out that Cauty's statement was false by constructing an $M_{1}$-space $X$ (cf. [4]) such that $(X, A)$ was not semi-canonical for some closed subset $A$ of $X$.

The purpose of this paper is to discuss the semi-canonical property in the product space $X \times I$ of a $T_{1}$ space $X$ with the unit closed interval $I$ and to reduce it to a property in $X$.

TheOREM 1. Let $X$ be a $T_{1}$ space. Then $(X \times I, X \times\{0\})$ is a semi-canonical pair if and only if $X$ is metrizable.

By Theorem 1 it can be easily seen that, if $X$ is any nonmetrizable $M_{1}$-space, then $X \times I$ is an $M_{1}$-space such that $(X \times I, X \times\{0\})$ is never semi-canonical.

Received by the editors November 8, 1976 and, in revised form, April 3, 1977.

AMS (MOS) subject classifications (1970). Primary 54E35; Secondary 54D30.

Key words and phrases. Semi-canonical cover, semi-canonical pair, $M_{1}$-space, metric space, compact-covering map, open map, $X$-base, countable character, first countable space.

() American Mathematical Society 1978 
Theorem 2. Let $X$ be a $T_{1}$ space. Then $(X \times I, K \times\{0\})$ is a semi-canonical pair for each compact subset $K$ if and only if $X$ is a regular space which is a compact-covering, ${ }^{1}$ open image of a metric space.

THEOREM 3. Let $X$ be a $T_{1}$ space. Then $(X \times I,\{(x, 0)\})$ is a semi-canonical pair for each point $x \in X$ if and only if $X$ is a regular, first countable space.

Throughout this paper, the following notations will be used: $X_{0}$ and $X_{n}$ denote the subspaces $X \times\{0\}$ and $X \times\{1 / n\}$ of $X \times I$ for $n=1,2, \ldots ; \pi$ denotes the projection from $X \times I$ onto $X$; and $I_{n}$ denotes the subspace $[0,1 / n]$ of $I$ for $n=1,2, \ldots$.

All spaces in this paper are $T_{1}$, and all maps are continuous.

2. Proof of Theorem 1. The sufficiency of the condition is clear, since every pair $(X, A)$ in a metric space $X$ is semi-canonical (cf. [6]). To prove necessity, suppose that there exists a semi-canonical cover $\mathcal{V}$ for $\left(X \times I, X_{0}\right)$. Put

$$
\mathscr{U}_{n}=\pi\left(\mathfrak{V} \mid X_{n}\right) \quad\left(=\left\{\pi\left(V \cap X_{n}\right): V \in \mathfrak{V}\right\}\right)
$$

for $n=1,2, \ldots$ Then $\left\{\mathscr{U}_{n}: n=1,2, \ldots\right\}$ is clearly a sequence of open covers of $X$.

Let us show that, for each point $x \in X$, the system $\left\{\operatorname{St}^{2}\left(x, \mathscr{U}_{n}\right): n=1\right.$, $2, \ldots\}$ forms a neighborhood base at $x$, where $\operatorname{St}^{2}(x, \mathscr{U})$ denotes the set $\operatorname{St}(\operatorname{St}(x, \mathcal{U}), \mathcal{U})$. Then $X$ is metrizable by a theorem of $\mathrm{K}$. Morita [10]. To complete the proof, let $x$ be any point of $X$ and $G$ an arbitrary neighborhood of $x$ in $X$. Since $\mathcal{V}$ is a semi-canonical cover for $\left(X \times I, X_{0}\right)$, there exist a neighborhood $H_{1}$ of $x$ in $X$ and a positive integer $m$ such that $\operatorname{St}\left(H_{1} \times I_{m}\right.$, V) $\subset G \times I$ holds. Again, for the neighborhood $H_{1}$ of $x$ there exist a neighborhood $\mathrm{H}_{2}$ of $x$ in $X$ and a positive integer $n$ such that $n \geqslant m$ and $\operatorname{St}\left(H_{2} \times I_{n}, \mathcal{V}\right) \subset H_{1} \times I$. Now, let us show $\operatorname{St}^{2}\left(x, \mathscr{U}_{n}\right) \subset G$. Pick an arbitrary point $y$ in $\operatorname{St}^{2}\left(x, \mathscr{U}_{n}\right)$. Then there are two members $U, U^{\prime}$ of $\mathscr{U}_{n}$ with $x \in U, y \in \cup^{\prime} U^{\prime}$ and $U \cap U^{\prime} \neq \varnothing$. Let $z$ be a point of $U \cap U^{\prime}$. By the definition of $\mathscr{U}_{n}$ there exist $V, V^{\prime}$ in $\mathfrak{V}$ such that

$$
U=\pi\left(V \cap X_{n}\right) \text { and } U^{\prime}=\pi\left(V^{\prime} \cap X_{n}\right) .
$$

Hence, $(x, 1 / n) \in V,(z, 1 / n) \in V \cap V^{\prime}$ and $(y, 1 / n) \in V^{\prime}$ hold. The first inclusion $(x, 1 / n) \in V$ implies $V \subset H_{1} \times I$, because $(x, 1 / n)$ belongs to $H_{2} \times I_{n}$; the second one implies $V^{\prime} \subset G \times I$, because $(z, 1 / n) \in V$ shows $(z, 1 / n) \in H_{1} \times I_{n}$ and $(z, 1 / n) \in V^{\prime}$ yields $V^{\prime} \cap\left(H_{1} \times I_{n}\right) \neq \varnothing$; and, as a consequence, the last inclusion $(y, 1 / n) \in V^{\prime}$ implies $y \in G$, which completes the proof.

\section{Some lemmas.}

LEMMA 1. Let $X$ be a space. If the pair $(X \times I,\{(x, 0)\})$ is semi-canonical for every point $x \in X$, then $X$ is a regular space.

${ }^{1}$ A continuous map $f: X \rightarrow Y$ is called compact-covering if every compact subset of $Y$ is the image of some compact subset of $X$. 
Proof. Using the same notations as in the proof of Theorem 1, it has been shown that, for a given point $x$ of $X$ and an arbitrary neighborhood $G$ of $x$, there exists an open cover $\mathscr{U}_{n}$ of $X$ such that $\operatorname{St}^{2}\left(X, \mathscr{Q}_{n}\right) \subset G$ holds. Clearly, $\operatorname{St}\left(x, \mathscr{U}_{n}\right)$ is a neighborhood of $x$, whose closure is contained in $\operatorname{St}^{2}\left(x, \mathscr{U}_{n}\right)$ and hence in $G$. This proves that $X$ is a regular space.

If $A \subset X$, then an $X$-base for $A$ is a collection $\mathscr{U}$ of open subsets of $X$ such that, if $x \in A$ and $V$ is a neighborhood of $x$ in $X$, then $x \in U \subset V$ for some $U \in$ Q.

LEMma 2. Let $X$ be a regular $\left(T_{1}\right)$ space and $K$ a compact subset of $X$. If there exists a countable $X$-base for $K$, then there exists an $X$-base $\cup_{n=1}^{\infty} \mathscr{P}_{n}$ for $K$ such that

(1) $\mathscr{P}_{\underline{n}}$ is a finite collection whose union covers $K$ for $n=1,2, \ldots$,

(2) $\left\{\stackrel{n}{P}: P \in \mathscr{P}_{n+1}\right\}$ refines $\mathscr{P}_{n}$ for $n=1,2, \ldots$, and

(3) for each point $x$ of $K$ and each neighborhood $G$ of $x$ in $X$, there exist a positive integer $n$ and a neighborhood $H$ of $x$ in $X$ such that $\operatorname{St}\left(H, \mathscr{P}_{n}\right) \subset G$.

Proof. Let $\mathscr{B}$ be the given countable $X$-base for $K$. Since $\mathscr{B} \mid K$ is a countable base for $K$ itself, $K$ is metrizable. Hence, for any subset $E$ of $K$, the diameter $\delta(E)$ of $E$ is well defined and also, for any cover $\mathcal{E}$ of $K$, the $\operatorname{mesh} \mathcal{E}=\sup \{\delta(E) ; E \in \mathcal{E}\}$ is well defined.

For each $n$, let $\mathscr{U}_{n}$ be a finite subcollection of $\mathscr{B}$ such that

(1) ${ }_{n} \mathscr{Q}_{n}$ covers $K$, and

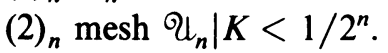

Let $\left\{\mathscr{V}_{n}: n=1,2, \ldots\right\}$ be the set of all finite subcollections of $\mathscr{B}$, each of which forms a minimal cover with respect to $K$; that is, any proper subcollection of $\mathcal{V}_{n}$ does not cover $K$ for $n=1,2, \ldots$ Put $\mathscr{W}_{1}=\mathcal{Q}_{1} \wedge \mathcal{V}_{1}$ $\left(=\left\{U \cap V: U \in \mathcal{U}_{1}, V \in \mathcal{V}_{1}\right\}\right)$ and $\mathscr{W}_{n+1}=\mathcal{W}_{n} \wedge \mathscr{U}_{n+1} \wedge \mathcal{V}_{n+1}$ for $n=$ $1,2, \ldots$ Then each $\mho_{n}$ is a finite collection of open subsets of $X$ whose union covers $K$.

Next, by induction on $n$, let us construct a finite collection $\mathscr{F}_{n}$ of closed subsets of $K$, a finite collection $\mathscr{P}_{n}$ of open subsets of $X$ and a function $\varphi_{n}$ from $\mathscr{F}_{n}$ onto $\mathscr{P}_{n}$ such that the following conditions are satisfied:

(3) ${ }_{n} \widetilde{F}_{n}$ is a closed cover of $K$ which refines $\mathscr{W}_{n} \wedge \mathscr{P}_{n-1}$, where $\mathscr{P}_{0}=\{X\}$,

(4) ${ }_{n} \mathscr{P}_{n}$ refines $\mathscr{W}_{n} \wedge \mathscr{P}_{n-1}$,

(5) ${ }_{n}$ if $F \in \mathscr{F}_{n}$, then $F \subset \varphi_{n}(F)$,

(6) $)_{n}$ if $F \in \mathscr{F}_{n}$ and $F \subset 0 \in \cup_{i=1}^{n-1} \mathscr{P}_{i} \cup \cup_{i=1}^{n}\left(\mathscr{U}_{i} \cup \mathscr{V}_{i}\right)$, then $\overline{\varphi_{n}(F)} \subset 0$, and

(7) $)_{n}$ if $F \cap F^{\prime}=\varnothing$, then $\varphi_{n}(F) \cap \varphi_{n}\left(F^{\prime}\right)=\varnothing$ for $F, F^{\prime} \in \mathscr{F}_{n}$.

Let $\mho_{1}=\left\{W_{1}, \ldots, W_{k}\right\}$. Since $\mho_{1}$ covers $K$ and $K$ is normal, there exists a closed cover $\mathscr{F}_{1}=\left\{F_{1}, \ldots, F_{k}\right\}$ of $K$ such that $F_{i} \subset W_{i}$ for $i=1, \ldots, k$. Hence $\mathscr{F}_{1}$ satisfies condition $(3)_{1}$. Since $X$ is regular and $\mathscr{F}_{1}$ is a finite collection, each member of which is compact, and since $\mathscr{Q}_{1}$ and $\mathfrak{V}_{1}$ are also finite collections, it is easy to see that the function $\varphi_{1}$ and $\mathscr{P}_{1}=\varphi_{1}\left(\mathscr{F}_{1}\right)$ are well defined to satisfy conditions $(4)_{1}-(7)_{1}$, as well. The situation in each step 
is the same as above, and thus $\mathscr{F}_{n}, \varphi_{n}$ and $\mathscr{P}_{n}$ are all constructed quite similarly.

Now, it remains to show that the sequence $\left\{\mathscr{P}_{n}: n=1,2, \ldots\right\}$ is the required one in Lemma 2 . Since $\mathscr{P}_{n}$ is finite and satisfies $(3)_{n}$ and (5) $)_{n}, \mathscr{P}_{n}$ satisfies the condition (1). By (3) $)_{n}$ and (6) $)_{n}, \mathscr{P}_{n}$ satisfies the condition (2). To prove that $\left\{\mathscr{P}_{n}: n=1,2, \ldots\right\}$ satisfies the condition (3), let $x$ be any point of $K$ and $G$ an arbitrary neighborhood of $x$ in $X$. Since $\mathscr{B}$ is an $X$-base for $K$, there exists a $B_{0} \in \mathscr{B}$ such that $x \in B_{0} \subset G{ }^{2}$ Let $\mathscr{V}$ be a finite subcollection of $\mathscr{B}$ which is a minimal cover with respect to $K$ and which keeps $B_{0}$ as the only member of $\mathcal{V}$ containing $x$. Since $K$ is a compact $T_{2}$ space and since $\mathscr{B}$ is an $X$-base for $K$, such $\mathscr{V}$ certainly exists; further, for some $n, \mathcal{V}=\mathfrak{V}_{n}$.

Let $F_{0} \in \mathscr{F}_{n}$ be a member with $x \in F_{0}$. Then $F_{0} \subset B_{0}$ holds, because $\mathscr{F}_{n}$ refines $\mathcal{W}_{n}$ which refines $\mathcal{V}_{n}$ and $B_{0}$ is the only member of $\mathcal{V}$ containing $x$; and also, by (5) ${ }_{n}$ and $(6)_{n}$, the inclusions $F_{0} \subset \varphi_{n}\left(F_{0}\right) \subset B_{0}$ hold. Since $\varphi_{n}\left(F_{0}\right)$ is an open set containing $x$, there exists a postive integer $m$ such that $m \geqslant n$ and $d\left(x, K-\varphi_{n}\left(F_{0}\right)\right)>1 / 2^{m}$, where $d$ denotes the metric function on $K$. Since $\mathscr{F}_{m+1}$ is a cover of $K$ by (3) $)_{m+1}$, there exists an $F_{1} \in \mathscr{F}_{m+1}$ containing $x$. To complete the proof, it suffices to show that

$$
\operatorname{St}\left(\varphi_{m+1}\left(F_{1}\right), \mathscr{P}_{m+1}\right) \subset \varphi_{n}\left(F_{0}\right),
$$

because $\varphi_{m+1}\left(F_{1}\right)$ is an open set in $X$ containing $x$ and $\varphi_{n}\left(F_{0}\right)$ is contained in $B_{0}$, which is contained in $G$. Let $P$ be an arbitrary member of $\mathcal{P}_{m+1}$ and $F$ the corresponding member of $\mathscr{F}_{m+1}$ by $P=\varphi_{m+1}(F)$. If $P \cap \varphi_{m+1}\left(F_{1}\right) \neq \varnothing$, then by $(7)_{m+1}, F \cap F_{1} \neq \varnothing$. Since $\mathscr{F}_{m+1}$ refines $\mathscr{P}_{m+1}$ by $(5)_{m+1}$ and $\mathscr{P}_{m+1}$ refines $\mathcal{W}_{m+1}$ by $(4)_{m+1}$, and since $\mathcal{W}_{m+1}$ refines $\mathcal{U}_{m+1}$ whose mesh restricting to $K$ is less than $1 / 2^{m+1}$, the diameter $\delta\left(F \cup F_{1}\right)$ is less than $1 / 2^{m}$. Since $x$ belongs to $F_{1}$, by the choice of $m, F \cup F_{1} \subset \varphi_{n}\left(F_{0}\right)$ holds. Again by (6) $m+1$, $\varphi_{m+1}(F) \subset \varphi_{n}\left(F_{0}\right)$ and thus $P \subset \varphi_{n}\left(F_{0}\right)$ holds, which completes the proof.

LEMma 3. Let $X$ be a regular $\left(T_{1}\right)$ space and $K$ a compact subset of $X$. If there exists a countable $X$-base for $K$, then $(X, K)$ is a semi-canonical pair.

Proof. Let $\cup_{n=1}^{\infty} \mathscr{P}_{n}$ be an $X$-base for $K$ obtained by Lemma 2 . For each $n$, put $G_{n}=\cup\left\{P: P \in \mathscr{P}_{n}\right\}$. Then, by conditions (1) and (2) in Lemma 2, $\bar{G}_{n+1} \subset G_{n}$ for $n=1,2, \ldots$ and $K \subset \cap_{n=1}^{\infty} G_{n}$, and by condition (3) and by the fact that $K$ is compact, it is easily seen that $K=\cap_{n=1}^{\infty} G_{n}$.

Now, put $\mathscr{V}_{0}=\left\{X-\bar{G}_{2}\right\}$ and $\mathscr{V}_{n}=\mathscr{P}_{n} \mid\left(G_{n}-\bar{G}_{n+2}\right)$ for $n=1,2, \ldots$, and put $\mathscr{V}=\cup_{n=0}^{\infty} \mathcal{V}_{n}$. Then it will be shown that $\mathcal{V}$ is a semi-canonical cover for $(X, K)$. Clearly, $\mathcal{V}$ is an open cover of $X-K$. To complete the proof, let $x$ be any point of $K$ and $U$ an arbitrary neighborhood of $x$ in $X$. By condition (3) in Lemma 2, there exist a positive integer $n$ and a neighborhood $H$ of $x$ in $X$ such that $\operatorname{St}\left(H, \mathscr{P}_{n}\right) \subset U$. Put $W=H \cap G_{n+1}$. Then $W$ is a neighborhood of $x$ in $X$ such that $W \cap V=\varnothing$ for each $V \in \cup_{i=1}^{n-1} \mathcal{V}_{i}$.

2 If $K$ is singleton, then $\bigcup_{n=1}^{\infty} \mathscr{P}_{n}$ is easily chosen from the given countable $X$-base for $K$, because $X$ is regular. So, assuming that $K$ is not a singleton, $B_{0}$ is picked out from $\mathscr{B}$ such that $K-B_{0} \neq \varnothing$. 
Therefore

$$
\operatorname{St}(W, \mathscr{V})=\operatorname{St}\left(W, \bigcup_{i>n} \mathscr{V}_{i}\right) \subset \operatorname{St}\left(W, \bigcup_{i>n} \mathscr{P}_{i}\right) \subset \operatorname{St}\left(H, \mathscr{P}_{n}\right) \subset U
$$

by condition (2) in Lemma 2, and that completes the proof.

4. Proofs of Theorems 2 and 3. The following characterization of the compact-covering open images of metric spaces, due to E. Michael and K. Nagami [9] will be used in the proof of Theorem 2.

Theorem M-N (E. Michael and K. NAgami). ${ }^{3}$ For a $T_{2}$ space $X$, the following conditions are equivalent:

(1) $X$ is the compact-covering open image of a metric space.

(2) Every compact subset of $X$ is metrizable and of countable character in $X{ }^{4}$

(3) Every compact subset of $X$ has a countable $X$-base.

Proof of Theorem 2. Necessity. Let $(X \times I, K \times\{0\})$ be a semi-canonical pair for any compact subset $K$ of $X$. Then $X$ is a regular space by Lemma 1 putting $K$ in the assumption a singleton. Next, it will be shown that each compact subset $K$ of $X$ has a countable $X$-base. Then $X$ is the compactcovering open image of a metric space by Theorem M-N.

To complete the proof, let $K$ be a compact subset of $X$. By the assumption, there exists a semi-canonical cover $\mathcal{V}$ for $(X \times I, K \times\{0\})$. Put $\mathfrak{V}_{n}$ the finite subcollection of $\mathcal{V}$ which covers $K \times\{1 / n\}$, and put $\mathscr{Q}_{n}=\pi\left(\mathscr{V}_{n} \mid X_{n}\right)$ for $n=1,2, \ldots$

Then it is easy to show that the collection $\cup_{n=1}^{\infty} \mathscr{U}_{n}$ is the required $X$-base for $K$, by the same technique as in the proof of Theorem 1 .

Sufficiency. It is easy to check that, if $X$ is the compact-covering open image of a metric space, then so is $X \times I$. Hence, for any compact subset $K$ of $X, K \times\{0\}$ has a countable $X \times I$-base by Theorem $\mathrm{M}-\mathrm{N}$, and thus $(X \times I, K \times\{0\})$ is a semi-canonical pair by Lemma 3, which completes the proof.

Proof of Theorem 3. Necessity. By Lemma $1, X$ is a regular space. The first countability of $X$ is proved by the same technique as in the proof of the necessity in Theorem 2 , replacing $K$ by a singleton.

Sufficiency. If $X$ is a regular $\left(T_{1}\right)$ first countable space, then so is $X \times I$. In general, it is easily seen that, in any regular $\left(T_{1}\right)$ first countable space $Y$, the pair $(Y,\{y\})$ is always semi-canonical for each point $y \in Y$. This completes the proof.

5. Comments. 1. From the proofs of Theorems 1, 2 and 3, it is easy to see that, in the conditions of these theorems, the closed interval $I$ may be

\footnotetext{
${ }^{3}$ The fact (2) $\rightarrow$ (3) was proved by M. M. Coban [5]; for completely regular space $X$, it had previously been obtained by A. V. Arhangel'skii [1].

${ }^{4}$ A set $K \subset X$ is of countable character in $X$ if there is a countable outer base $\left\{U_{n}: n=1\right.$, $2, \ldots\}$ at $K$ in $X$ (i.e. each $U_{n}$ is open and contains $K$, and every open set containing $K$ contains some $U_{n}$ ) (cf. [9]).
} 
replaced by any space containing a convergent sequence. By such replacement in Theorem 1, one obtains a slight modification of the proof of the following theorem due to D. M. Hyman [7], remembering two facts: (1) The closed image of a metric space is a Fréchet-Urysohn space (cf. [8]); and (2) any pair $(X, A)$ is semi-canonical if $X$ is the closed image of a metric space (cf. [7]).

TheOREM (D. HyMAN). If $X$ and $Y$ are nondiscrete spaces and if $X \times Y$ is the closed image of a metric space, then $X$ and $Y$ are metrizable.

2. The semi-canonical property need not be two-productive. For example, let $X=N \cup\{p\}$ be a subspace of Stone-Čech compactification $\beta N$ of $N$ $(=\{1,2, \ldots\})$ with $p \in \beta N-N$. Then it is well known that $X$ is not first countable at $p$, and thus $(X \times I,\{(p, 0)\})$ is not semi-canonical by Theorem 3. However, it is easy to see that any pair $(X, A)$ is always semi-canonical.

This example also shows that, in the conditions of Theorems 1 and 2, $X \times I$ cannot be replaced by $X$. Clearly, then, the semi-canonical property in $X$ is very different from the semi-canonical property in $X \times I$.

\section{REFERENCES}

1. A. V. Arhangel'skii, Bicompact sets and the topology of spaces, Trudy Moskov. Mat. Obšc. 13 (1965), 3-55 = Trans. Moscow Math. Soc. 13 (1965), 1-62.

2. C. J. R. Borges, On stratifiable spaces, Pacific J. Math. 17 (1966), 1-16.

3. M. R. Cauty, Une généralisation du théorème de Borsuk-Whitehead-Hanner aux espaces stratifiables, C. R. Acad. Sci. Paris Sér. A-B 275 (1972), A271-A274.

4. J. G. Ceder, Some generalizations of a metric space, Pacific J. Math. 11 (1961), 105-126.

5. M. M. Coban, Perfect mappings and spaces of countable type, Vestnik Moskov. Univ. Ser. I Mat. Meh. 22 (1967), no. 6, 87-93.

6. D. M. Hyman, A generalization of the Borsuk-Whitehead-Hanner theorem, Pacific J. Math. 23 (1967), 263-271.

7. D. M. Hyman, A note on closed maps and metrizability, Proc. Amer. Math. Soc. 21 (1969), 109-112.

8. N. Lasnev, Closed images of metric spaces, Dokl. Akad. Nauk SSSR $170(1966)=$ Soviet Math. Dokl. 7 (1966), 1219-1221.

9. E. Michael and K. Nagami, Compact-covering images of metric spaces, Proc. Amer. Math. Soc. 37 (1973), 260-266.

10. K. Morita, On the simple extension of a space with respect to a uniformity. IV, Proc. Japan Acad. 27 (1951), 632-636.

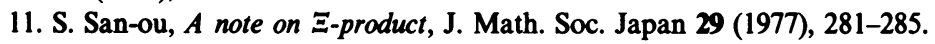

Department of Mathematics, Osaka Kyoiku University, Tennoj, Osaka, Japan 